\title{
New product-specific bioequivalence guidance
}

\author{
Christoph Baumgärtel, MD
}

\author{
For harmonization of the authorization requirements for specific generic applications within the EU, consistent criteria were drafted \\ for 16 active substances, which are out now for public consultation.
}

\section{Keywords: Authorization, bioequivalence, generics, product-specific bioequivalence guidance}

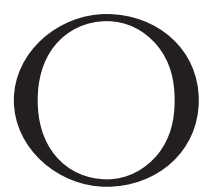

fficial draft guidance has recently become available for 16 new active substances that have either lost patent protection, will imminently lose it, or whose data-protection period has recently expired. The guidance establishes which criteria should be used when investigating the bioequivalence of a certain generic drug to its originator for these 16 substances.

The so-called product-specific bioequivalence guidance was published on 15 November 2013 [1], after development by the Pharmacokinetics Working Party (PKWP) of the European Medicines Agency (EMA), links to the published product-specific bioequivalence guidances (PSBEGS) can be found on the EMA website [2, 3].

By clearly stating which criteria should apply to these active substances, problems that have repeatedly led to questions and uncertainties during product development and application for marketing authorization can be avoided in the future.

Clearer guidance can sometimes resolve controversial issues: for example, establishing for distinct substances which strength of medicine is suitable and should be investigated in a bioequivalence trial; determining whether a study should be conducted in a fasted or fed state so that it is clear whether individuals participating in a trial should take medicines with food; or, instead of conducting a bioequivalence trial, a waiver using the biopharmaceutics classification system approach can be applied, which means that in vitro data will mainly suffice.

Product-specific bioequivalence guidance will make it clear for applicants whether the conventional bioequivalence criteria of $80-125 \%$ should apply, whether the $90-111 \%$ interval is required, or, in the case of highly variable drugs, a broadened acceptance margin of up to $70-143 \%$ may be chosen. Additionally, it will become clear if the parent of an active substance has to be measured in the plasma or the metabolite, or even both. It will also distinguish when it is appropriate to use healthy volunteers in a trial compared with patients.

The aim of product-specific bioequivalence guidance is to rationalize the criteria applied to substances in the authorization process, thereby giving companies more planning security when drafting an application, including the planning and carrying out of bioequivalence studies. It also gives the EU and all European national medicines authorities a single harmonized view on which criteria are deemed well founded and necessary for market authorization of generics with certain active substances.
Interested parties and stakeholders can forward comments on each of these active substances to pkwpsecretariat@ema.europa.eu on or before 15 February 2014.

The 16 published product-specific bioequivalence guidance substances are as follows:

- Capecitabine

- Carglumic acid

- Dasatinib

- Emtricitabine/Tenofovir

- Oseltamivir

- Posaconazole

- Repaglinide

- Erlotinib

- Imatinib

- Sirolimus

- Sorafenib

- Tadalafil

- Memantine

- Miglustat

- Telithromycin

- Voriconazole

As a member of EMA PKWP that drafted the guidance, I fully support this initiative, which is the first of its kind and highly essential for pharmaceutical companies. We are actively encouraging participation of stakeholders during the consultation process. Please use this opportunity to comment on the $16 \mathrm{draft}$ guidelines.

\section{Competing interests: None.}

Provenance and peer review: Commissioned; internally peer reviewed.

\section{References}

1. GaBI Online - Generics and Biosimilars Initiative. EMA releases productspecific bioequivalence guidelines [www.gabionline.net]. Mol, Belgium: Pro Pharma Communications International; [cited 2014 Jan 6]. Available from: www. gabionline.net/Guidelines/EMA-releases-product-specific-bioequivalenceguidelines

2. European Medicines Agency. EMA promotes consistent development of bioequivalence studies through product-specific guidance [homepage on the Internet]. 2013 Nov 11 [cited 2014 Jan 6]. Available from: http://www.ema. europa.eu/ema/index.jsp?curl=pages/news_and_events/news/2013/11/news_ detail_001958.jsp\&mid=WC0b01ac058004d5c1

3. European Medicines Agency. Clinical efficacy and safety: clinical pharmacology and pharmacokinetics [homepage on the Internet]. [cited 2014 Jan 6]. Available from: http://www.ema.europa.eu/ema/index.jsp?curl=pages/regulation/ general/general_content_000370.jsp\&mid=WC0b01ac0580032ec5

DOI: $10.5639 /$ gabij.2014.0301.009

Copyright $\odot 2014$ Pro Pharma Communications International

Author: Christoph Baumgärtel, MD, MSc, Department Head, Department Safety and Efficacy Assessment of Medicinal Products, Institute Marketing Authorisation of Medicinal Products \& LCM, AGES MEA-Austrian Medicines and Medical Devices Agency, and Austrian Federal Office for Safety in Health Care (BASG), European Expert in Pharmacokinetics Working Party of EMA, Member of Austrian Prescription Commission, 5 Traisengassee, AT-1200 Vienna, Austria

Submitted: 2 January 2014; Revised: 6 January 2014; Accepted: 7 January 2014; Published online first: 20 January 2014 